\title{
An ISO/SWS study of the dust composition around S stars ${ }^{\star}$ A novel view of S-star dust
}

\author{
S. Hony ${ }^{1}$, A. M. Heras ${ }^{2}$, F. J. Molster ${ }^{3}$, and K. Smolders ${ }^{4}$ \\ 1 Laboratoire AIM, CEA/DSM - CNRS - Université Paris Diderot, DAPNIA/Service d'Astrophysique, Bât. 709, CEA-Saclay, \\ 91191 Gif-sur-Yvette Cédex, France \\ e-mail: sacha.hony@cea.fr \\ 2 Research and Scientific Support Department-ESA/ESTEC, PO Box 299, 2200 AG Noordwijk, The Netherlands \\ 3 NOVA, PO Box 9513, 2300 RA Leiden, The Netherlands \\ 4 Instituut voor Sterrenkunde, K.U. Leuven, Celestijnenlaan 200D, 3001 Leuven, Belgium
}

Received 9 March 2009 / Accepted 22 April 2009

\begin{abstract}
Aims. We investigate the composition of the solid-state materials in the winds around S-type AGB stars. The S stars produce dust in their wind that bears a resemblance to the dust produced in some O-rich AGB stars. However, the reported resemblance is mostly based on IRAS/LRS spectra with limited spectral resolution, sensitivity, and wavelength coverage.

Methods. We investigate the dust composition around S stars using ISO/SWS data that surpass the previous studies in terms of spectral resolution and wavelength coverage. We selected the dust producing $\mathrm{S}$ stars in the ISO/SWS archive with enough signal to perform a detailed dust analysis, and then compare the dust spectra from the 9 sources with the O-rich AGB spectra and a subset of M super-giants. We constructed average dust emission spectra of the different categories.

Results. We report the discovery of several previously unreported dust emission features in the S star spectra. The long wavelength spectra of W Aql and $\pi^{1}$ Gru exhibit the " 30 " $\mu$ m feature attributed to MgS. Two sources exhibit a series of emission bands between 20 and $40 \mu \mathrm{m}$ that we tentatively ascribe to Diopside. We show that the 10-20 $\mu \mathrm{m}$ spectra of the $\mathrm{S}$ stars are significantly different from the O-rich AGB stars. The O-rich stars exhibit a structured emission feature that is believed to arise from amorphous silicate and aluminium-oxide. The S stars lack the substructure found in the O-rich stars. Instead they show a smooth peak with a varying peakposition from source to source. We suggest that this feature is caused by a family of related materials, whose exact composition determines the peak position. The observed trend mimics the laboratory trend of non-stoichiometric silicates. In this scenario the degree of non-stoichiometry is related to the $\mathrm{Mg}_{\mathrm{g}}$ to $\mathrm{SiO}_{4}$ ratio, in other words, to the amount of free $\mathrm{O}$ available during the dust grain growth.
\end{abstract}

Key words. stars: AGB and post-AGB - stars: mass-loss - infrared: stars - circumstellar matter - supergiants

\section{Introduction}

Asymptotic giant branch (AGB) stars are evolved stars of low to intermediate mass in the ZAMS mass range of $\sim 1$ to $8 M_{\odot}$. During the AGB phase, these stars are typified by high luminosity and low surface temperature, which implies a very large radius and low surface gravity. These stars often exhibit substantial mass loss through a dust driven or pulsation driven wind. This mass loss is important for several reasons. $i$ ) These winds provide the means through which these stars return nucleosynthesis products (i.e. metals) from the interior of the star into the interstellar medium (ISM). As the bulk of all stars fall in this mass range, the winds of AGB stars are one of the dominant contributors to the enrichment of the ISM. ii) These dusty winds alter the appearance of these stars because they cause the star to be surrounded by an envelope of gas and dust. The dust will absorb the stellar radiation and reradiate in the IR; therefore if one is to

\footnotetext{
* based on observations obtained with ISO, an ESA project with instruments funded by ESA Member states (especially the PI countries: France, Germany, the Netherlands and the United Kingdom) and with the participation of ISAS and NASA.
}

study the properties of these stars, the dusty envelope needs to be taken into account. iii) The further evolution of the star is determined by the mass loss. Unlike most types of stars, AGB star evolution is not determined by the nuclear fusion processes in the interior but by the mass loss at the surface and the AGB will end when the reservoir of envelope material has been exhausted by the mass loss.

The type of molecules and solid-state particles that are present in the winds are to the first order determined by the elemental abundances at the surface of the star. Most important in this respect is the number ratio of C-atoms to O-atoms ( $\mathrm{C} / \mathrm{O}$ ratio), because the carbon-monoxide ( $\mathrm{CO}$ ) molecule is easily formed and very stable. This causes most of the $\mathrm{C}$ - and $\mathrm{O}$-atoms to be present in the form of $\mathrm{CO}$. These atoms are then effectively not taking part in the chemistry and dust- condensation that occurs in these surroundings. Only the fraction of either $\mathrm{O}$-atoms if $\mathrm{C} / \mathrm{O}$ is smaller than unity, or $\mathrm{C}$-atoms if $\mathrm{C} / \mathrm{O}$ exceeds unity, is available. This dichotomy as a function of $\mathrm{C} / \mathrm{O}$ ratio is clearly found in the AGB stars. When $\mathrm{C} / \mathrm{O}<1$, i.e. in O-rich AGB stars, one finds large amounts of oxygenbearing molecules, like $\mathrm{SiO}, \mathrm{H}_{2} \mathrm{O}$ and $\mathrm{CO}_{2}$, while for $\mathrm{C} / \mathrm{O}>1$, 
i.e. in C-rich AGB stars, molecules like $\mathrm{CH}, \mathrm{C}_{2} \mathrm{H}_{2}$ and $\mathrm{HCN}$ are present. The same holds for the composition of the dust around these objects. The O-rich stars exhibit silicates and oxides like amorphous aluminium-oxide $\left(\mathrm{Al}_{2} \mathrm{O}_{3}\right)$ or spinel, while their $\mathrm{C}$-rich counterparts produce amorphous carbon, silicon carbide and sulfides like $\mathrm{MgS}$.

During the AGB phase the elemental abundances on the surface of the star are being altered by a process called dredgeup. This causes fusion products from the interior to be transported to the surface, gradually increasing the $\mathrm{C} / \mathrm{O}$ ratio. So, in the broadest possible terms stars on the AGB gradually evolve from O-rich $\mathrm{AGB}$ stars to $\mathrm{C}$-rich $\mathrm{AGB}$ stars. In this scenario the $\mathrm{S}$ stars form an interesting intermediate class of objects that have $\mathrm{C} / \mathrm{O} \simeq 1$. Simplemindedly, one would assume that in such surroundings the range of molecules and dust components could be very wide perhaps overlapping with both the typical $\mathrm{O}$-rich and C-rich species or species that are not found in either surroundings. Theoretically, iron-silicide and metallic iron are the dust species predicted on the basis of chemical equilibrium calculations (Ferrarotti \& Gail 2002). Observationally, this class of objects has not been exhaustively studied to determine the dust composition although some studies have focused on the $10 \mu \mathrm{m}$ emission feature from S stars (Little-Marenin \& Little 1988; Chen \& Kwok 1993; Sloan \& Price 1998; Speck et al. 2000). In particular the spectra obtained with the Short Wavelength Spectrometer (SWS) (de Graauw et al. 1996) onboard the Infrared Space Observatory (ISO) (Kessler et al. 1996) which cover a much broader wavelength range $(2-45 \mu \mathrm{m})$ than available before with a high sensitivity and spectral resolving power, allow us to get a more complete picture of the composition around $\mathrm{S}$ stars.

The most diagnostic feature in the $\mathrm{S}$ star spectra is found in the $10 \mu \mathrm{m}$ region. The dust emission features in the $10 \mu \mathrm{m}$ region are very extensively studied because $i$ ) this window is available for ground-based observations; $i$ ) it holds the important diagnostic resonances of both $\mathrm{O}$-rich (silicates, $\mathrm{Al}_{2} \mathrm{O}_{3}$ and spinel) and $\mathrm{C}$-rich ( $\mathrm{SiC}$ ) dust and iii) spectra for many evolved stars in this region are available in the form of IRAS/LRS spectra. Many studies have focused on classifying the spectral appearance of the $\sim 10 \mu \mathrm{m}$ emission band, using various classification schemes, and relating the classification to the other observable characteristics of the sources like the spectral type, luminosity class, the $\mathrm{C} / \mathrm{O}$ ratio, the variability type or the massloss rate (Little-Marenin \& Little 1988; Skinner et al. 1990; Little-Marenin \& Little 1990; Chen \& Kwok 1993; Sloan \& Price 1995, 1998; Sylvester 1999; Speck et al. 2000; Miyata et al. 2000; Kraemer et al. 2002; Yang et al. 2007). Here we do not repeat the details of these studies but focus on the main conclusions that are relevant for the current work and refer to Sloan \& Price (1998) and Speck et al. (2000) for extensive and thorough comparisons of the relevant literature and the various interpretations of the dust emission characteristics.

The main points that have emerged are as follows:

1. O-rich AGB stars show a range of dust emission features with a range of peak positions.

(a) A single narrow peak at $9.7 \mu \mathrm{m}$ (on a $F_{v}$ scale), due to "classical silicates". The corresponding Si-O bending mode resonance is also observed near $18 \mu \mathrm{m}$.

(b) A broader structured feature, with local maxima at 9.7, 11 and $13 \mu \mathrm{m}$, with the peak-position close to $\sim 10 \mu \mathrm{m}$.

(c) A broad and shifted feature peaking at longer wavelength (up to $13 \mu \mathrm{m}$ ). This broad feature exhibits the same local maxima as mentioned above. It is generally accepted that this broad feature is due to the dominant contribution of dust components other than the silicates, probably amorphous aluminium-oxide (Onaka et al. 1989).

The origin of the structured features (b) in between the two extremes above is uncertain. While Onaka et al. (1989) and Lorenz-Martins \& Pompeia (2000) model these spectra with a mixture of silicates and aluminium-oxide, Egan \& Sloan (2001) find that these observations, with the additional constraints set by the IRAS broadband photometry, are best explained using pure silicates in a dust shell of larger optical depth. However, the fact that one finds the same substructures within a wide range of peak-positions argues against the opacity effect being dominant, and most likely these sources represent a mixture of silicate and aluminium-oxide grains.

2. M super-giants exhibit a similar range of $10 \mu \mathrm{m}$ features, although they are more weighted to the classical silicate profiles (a) and the broad (c)-type features are rare (Sloan \& Price 1998). An interesting exception is a significant fraction of $\mathrm{M}$ super-giants of the $\mathrm{h}$ and $\chi$ Per association that exhibit a (c)-type feature peaking near $10.5 \mu \mathrm{m}$ with the $11.3 \mu \mathrm{m}$ UIR band perched on top (Sylvester et al. 1998). In Sect. 5 we will compare these interesting sources with the S stars.

3. Little-Marenin \& Little (1988) find that S-type stars preferentially exhibit a broad feature peaking in the $10.5-10.8 \mu \mathrm{m}$ range, which in fact makes them dub this feature the " $\mathrm{S}$ " feature. This agrees well with the findings of Sloan \& Price (1998), who also find that the S-type stars in their sample predominantly exhibit such a broad feature. In contrast Chen \& Kwok (1993) report that the S-type stars have emission features very similar to $M$ stars, covering the complete range from pure silicates to the broad feature profile. This difference is easily understood from the fact that the latter authors include in their much larger sample also many MS and weak $\mathrm{S}$ stars which have a $\mathrm{C} / \mathrm{O}$ ratio well below unity and should have dust similar to the $\mathrm{M}$ stars. The profile found in the strong $\mathrm{S}$ stars is broad and peaking in the 10.5-10.8 $\mu \mathrm{m}$ range. The extend and peak-position of this $S$ star feature are very similar to the broad features found in the O-rich AGB stars, however the $13 \mu \mathrm{m}$ feature is lacking in the $\mathrm{S}$ star spectra (Sloan \& Price 1998, Fig. 9). These authors also find that there might be a slight enhancement between 10 to $11 \mu \mathrm{m}$ in the S-type feature when compared to the $\mathrm{M}$ star sample, although they state that the difference might not be significant given the quality of the LRS spectra they used.

In this paper we explore the dust composition around $\mathrm{S}$ stars as derived from the available SWS spectra.

\section{Observations}

The ISO/SWS database contains $22 \mathrm{~S}$ star spectra that cover the full wavelength range of $2.3-45 \mu \mathrm{m}: 21 \mathrm{ISO} / \mathrm{SWS}$ sources listed in the second edition of the general catalogue of $S$ stars (Stephenson 1994) with the addition of the S star OP Her are given in Table 1. Of these 22 sources we include nine in the $S$ star sample that we explore here; NO Aur is a super-giant, II Lup is carbon-rich, ST Her is an MS star and T Cet is of uncertain nature. The other stars have either no significant dust emission or too little signal-to-noise to investigate their dust composition to the level of detail required here. 
Table 1. Details of the sources/SWS spectra used in this study.

\begin{tabular}{|c|c|c|c|c|c|c|c|}
\hline Source & IRAS name & $\mathrm{AOT}^{a}$ & $\mathrm{TDT}^{b}$ & Class. $^{c}$ & SP98 $^{d}$ & Spec. Type Ty $^{e}$ & Remarks \\
\hline \multicolumn{8}{|c|}{ S stars from Stephenson (1994) used in this study } \\
\hline R And & $00213+3817$ & $01(2)$ & 40201723 & 2.SEc & SE3 & $\mathrm{S} 3,5-8,8 \mathrm{e}$ & $18 \mu \mathrm{m}$ feat. weak \\
\hline $\mathrm{S}$ Cas & $01159+7220$ & $01(2)$ & 41602133 & 3.SEp & SE3 & $\mathrm{S} 3,4-5,8 \mathrm{e}$ & $15 \mu \mathrm{m}$ feat., $18 \mu \mathrm{m}$ feat. absent, $\mathrm{C}_{2} \mathrm{H}_{2}+\mathrm{HCN}$ abs. \\
\hline W Aql & $19126-0708$ & $01(2)$ & 16402335 & 3.SEp & SE3 & $\mathrm{S} 3,9-6,9 \mathrm{e}$ & $15 \mu \mathrm{m}$ feat., $18 \mu \mathrm{m}$ feat. absent, $\mathrm{C}_{2} \mathrm{H}_{2}+\mathrm{HCN}$ abs. \\
\hline R Cyg & $19354+5005$ & $01(1)$ & 42201625 & 2.SEb & SE3 & $\mathrm{S} 2.5,9-6,9 \mathrm{e}$ & $18 \mu \mathrm{m}$ feat. absent, $10 \mu \mathrm{m}$ feat. narrow \\
\hline$\chi$ Cyg & $19486+3247$ & $01(2)$ & 15900437 & 2.SEb & SE3 & S6, 2-10, 4e & $18 \mu \mathrm{m}$ feat. absent, substructure at $10.2 \mu \mathrm{m}$ \\
\hline AA Cyg & $20026+3640$ & $01(2)$ & 36401817 & 2.M & $\mathrm{N}$ & S7, 5-7.5, 6 & : \\
\hline RZ Sgr & $20120-4433$ & $01(2)$ & 14100818 & 2.SEa & SE2 & $\mathrm{S} 4,4 \mathrm{ep}$ & \\
\hline$\pi^{1} \mathrm{Gru}$ & $22196-4612$ & $01(2)$ & 34402039 & 2.SEa & SE2 & $\mathrm{S} 7,5 \mathrm{e}$ & $18 \mu \mathrm{m}$ feat. absent \\
\hline RX Lac & $22476+4047$ & $01(1)$ & 78200427 & 2.SEa & SE1 & $\mathrm{M} 7.5 \mathrm{Se}$ & \\
\hline \multicolumn{8}{|c|}{ Stars from Stephenson (1994) not included in the S star sample } \\
\hline W Cet & $23595-1457$ & $01(2)$ & 37802225 & 2.SEa: & - & $\mathrm{S} 7^{e 1}$ & Low SNR \\
\hline T Cet & 00192-2020 & $01(2)$ & 55502308 & 2.SEa & SE1t & M5-6S IIe & M supergiant, $\mathrm{M}, \mathrm{MS}$ or $\mathrm{S}$ \\
\hline RW And & $00445+3224$ & $01(3)$ & 42301901 & 7 & SE3: & M5-10e(S6,2e) & Low SNR \\
\hline WX Cam & $03452+5301$ & $01(2)$ & 81002721 & 1.NO & - & $\mathrm{S} 5 / 5.5^{e 2}$ & Low SNR \\
\hline NO Aur & $05374+3153$ & $01(1)$ & 86603434 & 2.SEa & SE1 & M2S Iab & Supergiant \\
\hline LY Mus & $13372-7136$ & $01(2)$ & 13201304 & $1 . \mathrm{NO}$ & - & M4III ${ }^{e 1}$ & No dust features \\
\hline II Lup & $15954-5114$ & 06 & 29700401 & 3. $\mathrm{CE}^{c 1}$ & - & $\mathrm{SC}^{e 3}$ & Carbon-rich \\
\hline ST Her & $15492+4837$ & $01(3)$ & 41901305 & 2.SEa & SE1 & M6-7 IIIaS & MS \\
\hline OP $\operatorname{Her}^{f}$ & $17553+4521$ & $01(1)$ & 77800625 & $1 . \mathrm{NO}$ & $\mathrm{N}$ & M5 IIb-IIIa(S) & No dust features \\
\hline HD 165774 & $18058-3658$ & $01(2)$ & 14100603 & 1.NO: & - & $\mathrm{M} 2 \mathrm{II} / \mathrm{III}^{e 1}$ & No dust features, low SNR \\
\hline S Lyr & $19111+2555$ & $01(1)$ & 52000546 & 2.CE: & SE2 & $\mathrm{SCe}$ & Low SNR \\
\hline HR Peg & $22521+1640$ & $01(2)$ & 37401910 & 1.NO & - & $\mathrm{S} 5,1^{e 1}$ & No dust features \\
\hline GZ Peg & $23070+0824$ & $01(3)$ & 37600306 & 1.NO & $\mathrm{N}:$ & M4S III & No dust features \\
\hline \multicolumn{8}{|c|}{ Supergiants with $10 \mu \mathrm{m}$ features resembling the $\mathrm{S}$ stars } \\
\hline KK Per & $02068+5619$ & $01(1)$ & 45701204 & 2.SEa & - & M2 Iab & $18 \mu \mathrm{m}$ feat. weak, UIR \\
\hline V605 Cas & $02167+5926$ & $01(2)$ & 61301202 & 2.SEa: & - & $\mathrm{M} 2 \mathrm{Iab}^{e 4}$ & $18 \mu \mathrm{m}$ feat. weak, UIR \\
\hline AD Per & $02169+5645$ & $01(2)$ & 78800921 & 2.SEap & - & $\mathrm{M} 2.5 \mathrm{Iab}^{e 5}$ & $18 \mu \mathrm{m}$ feat. weak, UIR \\
\hline NO Aur & $05374+3153$ & $01(1)$ & 86603434 & 2.SEa & SE1 & M2S Iab & $18 \mu \mathrm{m}$ feat. weak, UIR \\
\hline V1749 Cyg & $20193+3527$ & $01(2)$ & 73000622 & 2.SEb & SE3t & M3 Iab & UIR \\
\hline IRC +40427 & $20296+4028$ & $01(3)$ & 53000406 & 2.SEap: & - & M0-2 $\mathrm{I}^{e 6}$ & $18 \mu \mathrm{m}$ feat. absent, UIR \\
\hline CIT 11 & $20377+3901$ & $01(1)$ & 40503119 & 2.SEb & - & M3: Iab & $18 \mu \mathrm{m}$ feat. weak \\
\hline V354 Cep & $22317+5838$ & $01(2)$ & 41300101 & 2.SEc & SE6 & M2.7 Iab & $15 \mu \mathrm{m}$ feat., $18 \mu \mathrm{m}$ feat. absent \\
\hline V582 Cas & $23278+6000$ & $01(1)$ & 38501620 & 2.SEc & SE5 & $\mathrm{M} 4 \mathrm{I}^{e 7}$ & $18 \mu \mathrm{m}$ feat. weak, UIR: \\
\hline \multicolumn{8}{|c|}{ Other sources used in this study } \\
\hline IRC+50 096 & $03229+4721$ & $01(2)$ & 81002351 & 3.CE & - & $\mathrm{C}^{e 2}$ & Carbon-rich, " 30 ” $\mu \mathrm{m}$ feature \\
\hline R Hya & $13269-2301$ & $01(1)$ & 08200502 & 2.SEa & SE2t & M6-9eS(Tc) & \\
\hline TY Dra & $17361+5746$ & $01(2)$ & 74102309 & 2.SEc & SE8t & M5-8 & \\
\hline
\end{tabular}

${ }^{a}$ Observing mode used (see de Graauw et al. 1996; Clegg et al. 1996). Numbers in brackets correspond to the scanning speed. ${ }^{b}$ TDT number which uniquely identifies each ISO observation. ${ }^{c}$ Classification of the SWS spectrum from Kraemer et al. (2002), except ${ }^{c 1}$ Sloan et al. (2003). ${ }^{d}$ IR classification in the scheme of Sloan \& Price (1998). ${ }^{e}$ Spectral types are from Sloan \& Price (1998); except ${ }^{e 1}$ Kharchenko (2001); ${ }^{e 2}$ Buscombe (2001); ${ }^{e 3}$ Buscombe (1998); ${ }^{e 4}$ Humphreys (1970); ${ }^{e 5}$ Blanco (1955); ${ }^{e 6}$ Solf (1978) and ${ }^{e 7}$ Winfrey et al. (1994). ${ }^{f}$ This source is only listed in the first edition of general catalogue of S stars (Stephenson 1995) and is classed M star in the second edition.

\subsection{Data reduction}

The data were processed using SWS interactive analysis, IA (see de Graauw et al. 1996), using calibration files and procedures equivalent to pipeline version 10.1. Further data processing consisted of extensive bad data removal and rebinning on a fixed resolution $(\lambda / \Delta \lambda=200)$ wavelength grid. In order to combine the different sub-bands into one continuous spectrum from 2 to $45 \mu \mathrm{m}$ we applied scaling factors. In general the match between the different sub-bands is good and the applied scaling/offsets are small compared to the flux calibration uncertainties with a few exceptions:

\subsection{1. $\chi$ Cyg}

The band $2 \mathrm{~A}(4.1-5.3 \mu \mathrm{m})$ and $2 \mathrm{C}(7.0-12.5 \mu \mathrm{m})$ data are affected by strong memory effects. The shape of the up and downs scan of band $2 \mathrm{~A}$ differs. The slope of the band $2 \mathrm{C}$ data differs while the details of the shape and substructure are present in both scans. The data appear to be also slightly affected by miss-pointing as we have to apply a scaling of $\sim 1.08$ to the band $1(2.4-4.1 \mu \mathrm{m})$ data and 1.2 to the band $3(12.5-29 \mu \mathrm{m})$ data.

\subsection{2. $\pi^{1}$ Gru}

The data for $\pi^{1}$ Gru are apparently affected by a slight misspointing and the data for the sub-bands $2 \mathrm{~A}$ to $3 \mathrm{D}(4.1-27.5 \mu \mathrm{m})$ need to be multiplied by a relatively large factor $(\sim 1.25)$ to be consistent with the flux levels at shorter and longer wavelengths. We note that the main signature of the $\mathrm{MgS}$ feature is contained within sub-band 3D $(19.5-27 \mu \mathrm{m})$ and is not affected by the scaling (see also Fig. 2). 


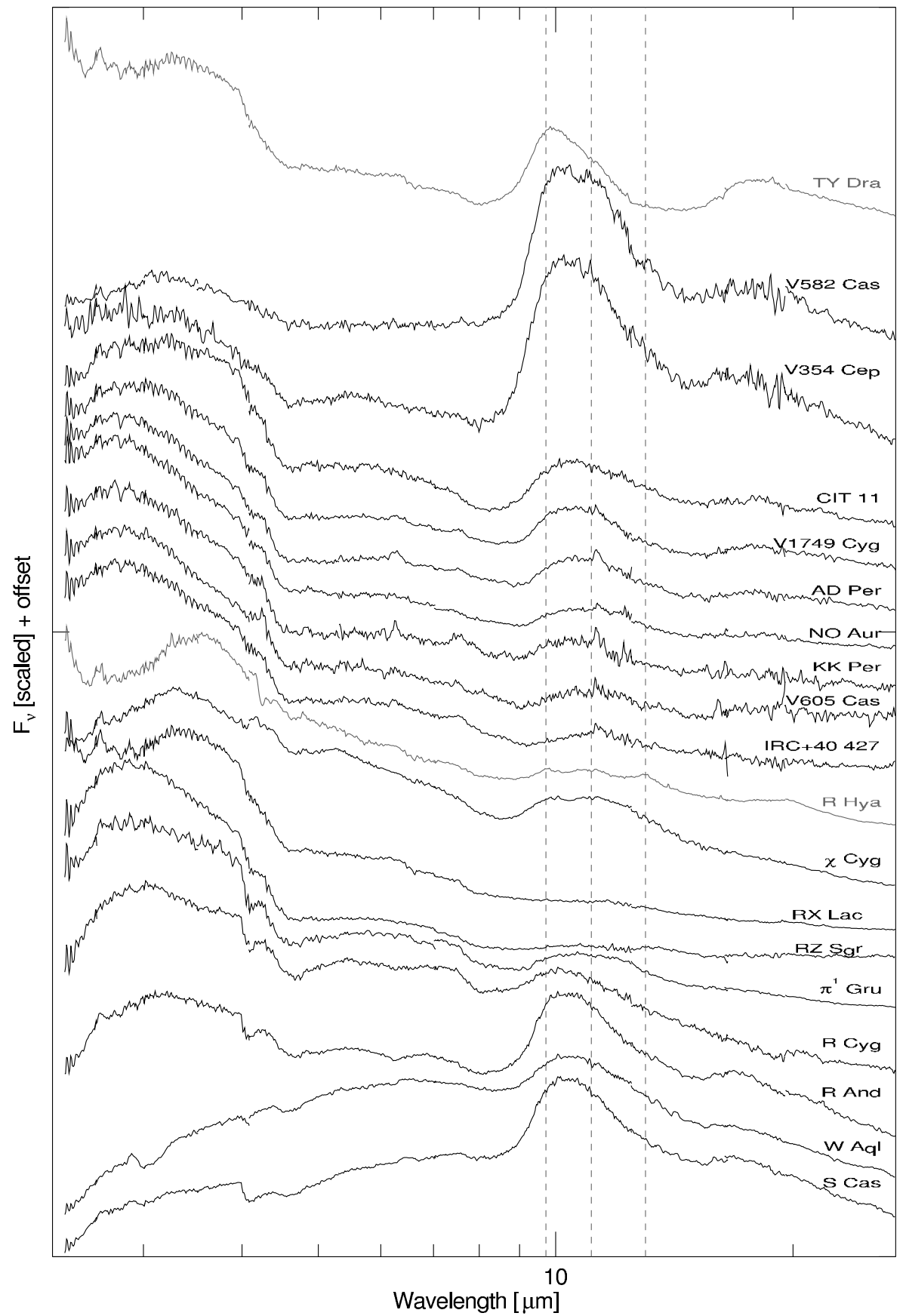

Fig. 1. ISO/SWS spectra of the sources listed in Table 1. From the bottom to the top we show. Eight spectra of S stars (S Cas $-\chi \mathrm{Cyg}$ ). The grey spectrum in the middle is R Hya, an O-rich AGB star that shows the typical structured $10 \mu \mathrm{m}$ feature. Then nine M super-giants that exhibit a smooth and displaced $10 \mu \mathrm{m}$ features (IRC+40 427 - V582 Cas). At the very top we show TY Dra as an example of classical silicate dominated spectrum. The dashed lines represent the wavelengths of the substructures found in the O-rich AGB stars.
The final reduced spectra are presented in Fig. 1. As it turns out these stars are very rich in their dust emission spectra exhibiting a wide range of dust features from different types of materials. We will first discuss the long wavelength part of the spectra of $\pi^{1}$ Gru, W Aql and RX Lac as they show features that have not been detected before around S stars.

\section{Long wavelength spectra}

\subsection{The "30 $\mu m$ " feature}

In Fig. 4 we show the spectra of $\pi^{1}$ Gru and W Aql. We also show for comparison the SWS spectrum of the C-rich red giant IRC +50 096, which exhibits purely C-rich dust features and the MS star ST Her, which exhibits O-rich dust features.
The most remarkable feature in the spectra of these two $\mathrm{S}$ stars is the emission feature starting at $23.5 \mu \mathrm{m}$ and peaking at $26 \mu \mathrm{m}$, indicated in Fig. 4 with $\mathrm{MgS}$. The feature is weak and even in the closeup of the region in the right panel of Fig. 5 not very prominent. This is probably also the reason why it has eluded detection until now.

In Fig. 2 we display the spectra in intensity units that better reveal the structure in this region. The shape of the feature found in the $S$ star spectra closely corresponds to the shape of the much stronger $\mathrm{MgS}$ resonance found in the C-rich star IRC +50 096. In particular the change of slope from 22 to $24 \mu \mathrm{m}$ and the second change beyond $26 \mu \mathrm{m}$ is detected in the three sources at the top. We also show the expected spectrum from $\mathrm{MgS}$, which exhibits the same shape. The two independent scans in the SWS data yield the same shape, attesting to the reality of the $\mathrm{MgS}$ detection. 


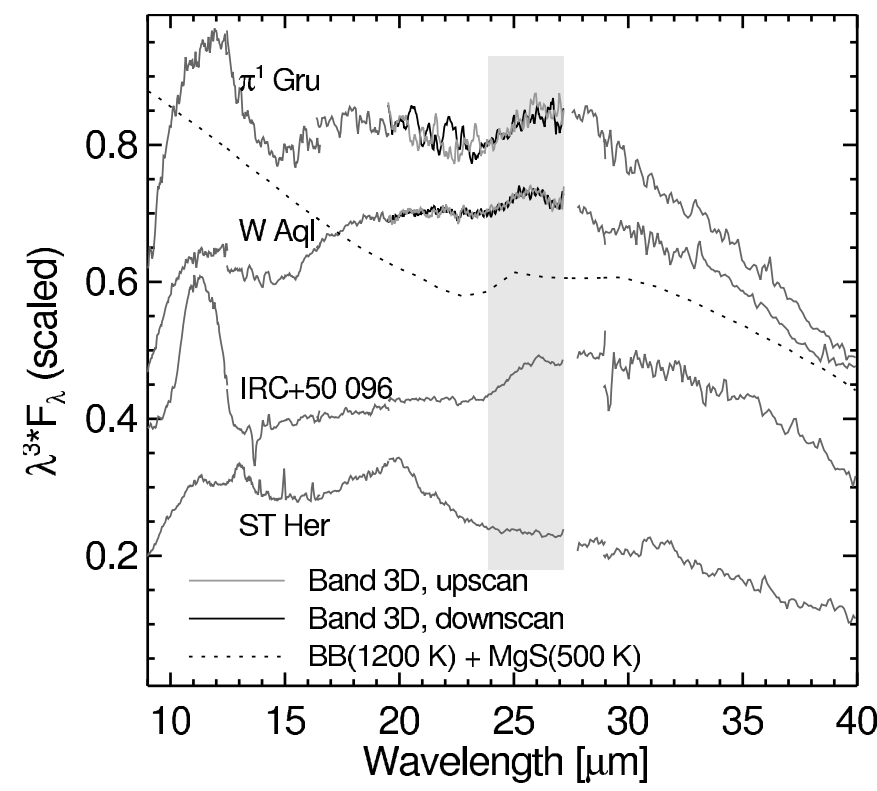

Fig. 2. Comparison of the features found in the SWS spectra of $\pi^{1}$ Gru and $\mathrm{W}$ Aql to the MgS feature in IRC+50 096. We show the signal of the two independent scans of sub-band 3D. The characteristic sharp rise from 24 to 26 (indicated in the light shaded area) with a gradual decline towards longer wavelength is found in all available scans. The scale along the ordinate is chosen to better bring out the structure in this wavelength domain without having to resort to removing an underlying baseline. The dotted line shows the spectral signature expected from a star surrounded by MgS, simulated with a Planck-function of $1200 \mathrm{~K}$ plus the emission of $\mathrm{MgS}$ grains at $500 \mathrm{~K}$ in a CDE-shape distribution. Near the bottom we show the spectrum of the MS star ST Her as an example of a source that does not show this feature.

\subsection{RX Lac and AA Cyg}

In Fig. 3 we show the spectra of RX Lac and AA Cyg. Both sources exhibit structure at 20,32.5 and $40 \mu \mathrm{m}$. The structure around $20 \mu \mathrm{m}$ seems to be quite secure in both spectra. The long wavelength part of the AA Cyg spectrum is very noisy. Comparing the detected features with available laboratory spectra they seem to correspond best to Diopside. There might be a connection between the appearance near $10 \mu \mathrm{m}$ as both sources exhibit a weak, very red emission feature and strong $\mathrm{SiO}$ absorption. This absorption, in combination with a low Diopside temperature, might explain why we do not detect the corresponding $10 \mu \mathrm{m}$ bands of Diopside. In should be borne in mind that the long wavelength spectra of these stars are quite noisy and that the correspondence with the laboratory spectrum is not perfect. In particular the $25 \mu \mathrm{m}$ feature which is present in the laboratory spectrum is markedly absent in the stellar emissions. Therefore this should be considered a tentative identification.

\section{The broad feature in the SWS spectra of S stars}

The most prominent dust feature in the spectra of these $\mathrm{S}$ stars is the broad and smooth emission feature centred around $\sim 11 \mu \mathrm{m}$ (Fig. 4 and the middle panel of Fig. 5). A similar feature (covering the same wavelength range) is found in the IR spectra of many O-rich evolved stars (e.g. Gillett et al. 1968; Little-Marenin \& Little 1990; Kraemer et al. 2002), which it is usually attributed to a combination of silicates and aluminiumoxide with the possible addition of spinel at $13 \mu \mathrm{m}$ (e.g. Woolf \& Ney 1969; Hackwell 1972; Posch et al. 1999; Cami 2002).

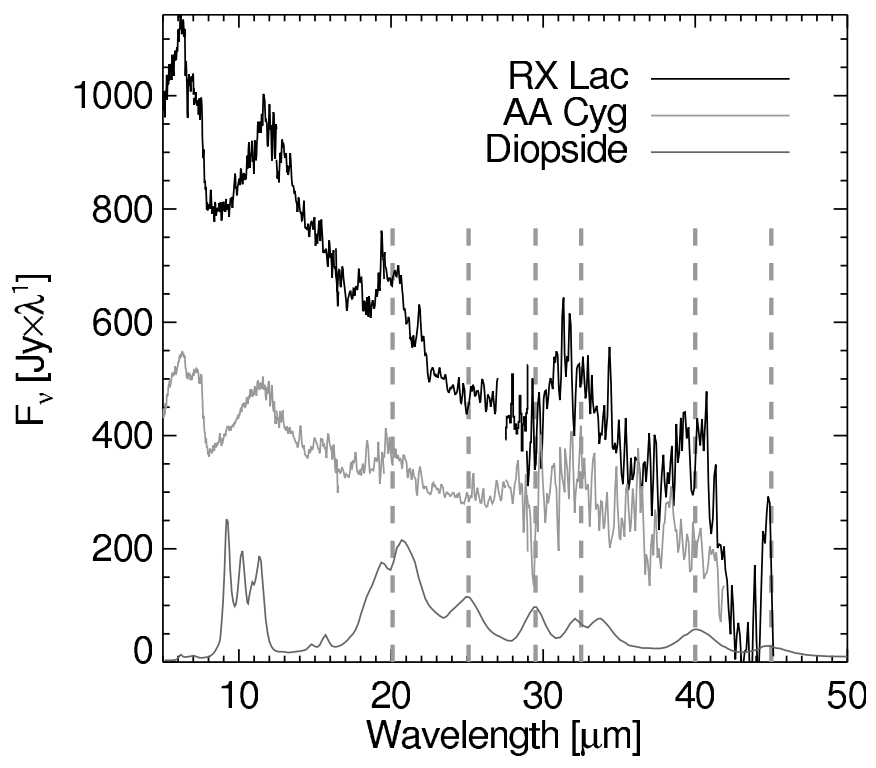

Fig. 3. Long wavelength spectra of RX Lac and AA Cyg. The intensity units are chosen to better exhibit the emission bands on the steeply dropping stellar continuum. Below we show the absorption cross-sections of Diopside ( $\mathrm{MgCaSi}_{2} \mathrm{O}_{6}$, Koike et al. 2000).

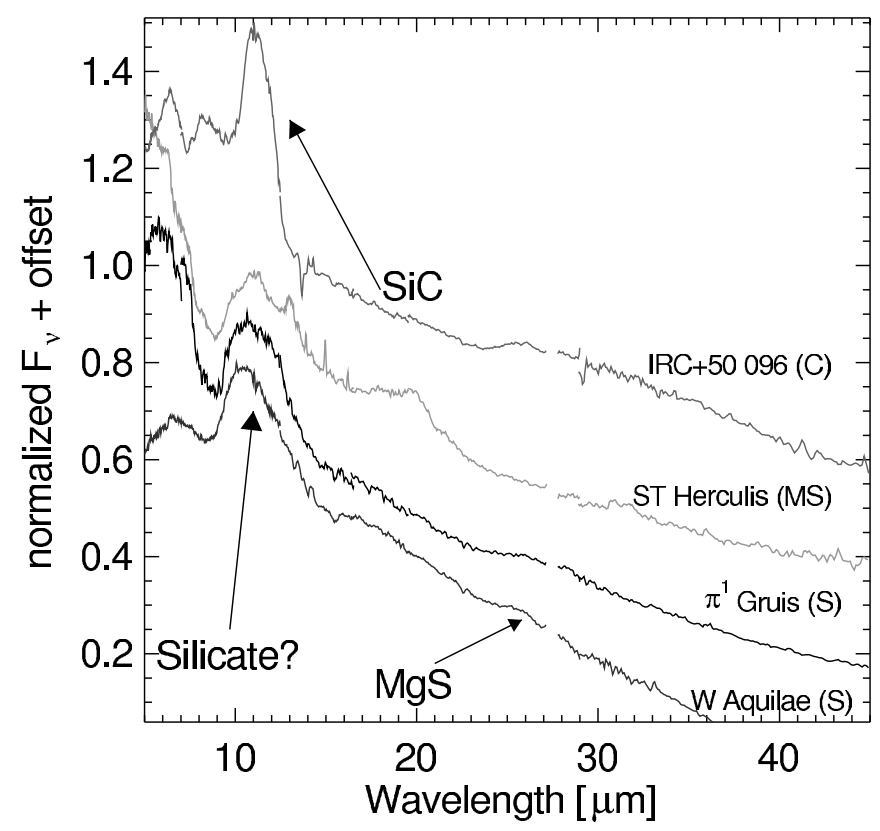

Fig. 4. Overview of the SWS spectra of four red giant stars. We show from top to bottom the carbon-rich AGB star IRC+50 096, the M6.5S star ST Her and the S stars $\pi^{1}$ Gru and W Aql. The carbon-rich star exhibits the typical $\mathrm{SiC}$ feature at $11.3 \mu \mathrm{m}$ and the $\mathrm{MgS}$ feature near $26 \mu \mathrm{m}$. The ST Her spectrum is dominated by the silicate dust features at 10 and $20 \mu \mathrm{m}$ which are typical for oxygen-rich environments. $\pi^{1}$ Gru and W Aql exhibit the MgS feature (see also Figs. 5 and 2) and a broad feature around $10 \mu \mathrm{m}$ which resembles the silicate emission but is lacking clear evidence of the corresponding $20 \mu \mathrm{m}$ silicate band. The latter is especially clear in the spectrum of $\pi^{1}$ Gru, while there is a weak feature near $\sim 17 \mu \mathrm{m}$ in the spectrum of $\mathrm{W}$ Aql.

However, the corresponding silicate feature due to the $\mathrm{Si}-\mathrm{O}$ stretch/bend near $18 \mu \mathrm{m}$ is remarkably weak.

The similarities between the S star $10 \mu \mathrm{m}$ features and the broad feature found in the O-rich spectra have until now been interpreted as the $\mathrm{S}$ stars showing silicate plus aluminium-oxide 

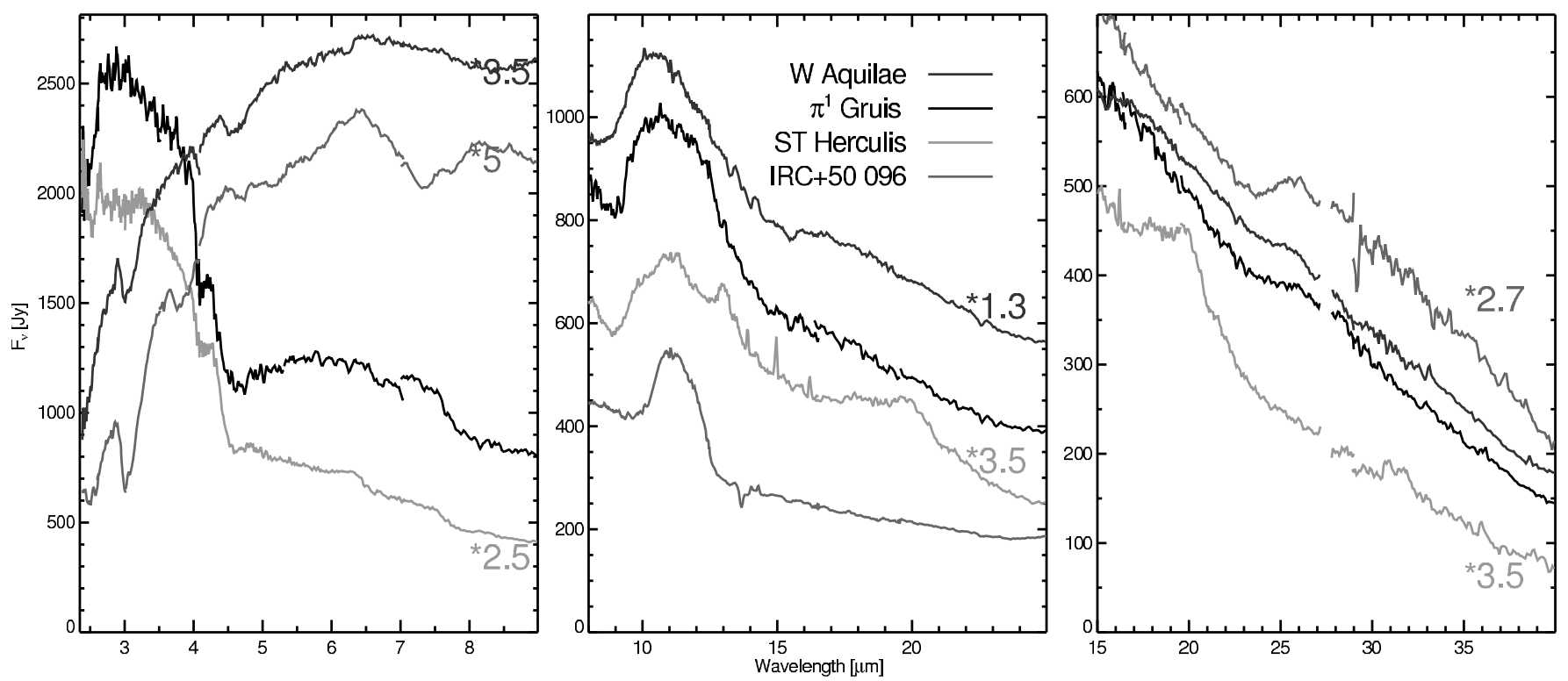

Fig. 5. Detailed view of the IR spectra of the stars presented in Fig. 4. The left panel shows the shortest wavelength region in which the spectral structure is dominated by molecular absorption bands. Clearly the molecular composition of $\pi^{1}$ Gru resembles that of ST Her. The typical C-rich molecular bands due to $\mathrm{C}_{2} \mathrm{H}_{2}, \mathrm{HCN}$ and $\mathrm{C}_{3}$ are absent in $\pi^{1}$ Gru but are seen in the IRC+50 096 and W Aql. The middle panel compares the 10 to $20 \mu \mathrm{m}$ range of these sources. The wavelength range of the broad $\sim 10 \mu \mathrm{m}$ emission band in the $\mathrm{S}$ star spectra is identical to the silicate emission of ST Her, however the feature appears more smooth and the sharp substructures at $9.5,10.8$ and $13 \mu \mathrm{m}$ are absent. Likewise, the $20 \mu \mathrm{m}$ silicate band is not observed. The silicon-carbide feature seen in IRC+50 096 corresponds in peak position but is much narrower than the emission feature in ST Her. Finally, the panel on the right shows the MgS emission feature which is detected in the IR spectra of $\pi^{1}$ Gru, W Aql and IRC +50096 and not in ST Her.

emission like the O-rich stars. In the following we will argue against this interpretation. Instead, we will show that there is a significant difference between the $\mathrm{M}$ star broad features due to silicates plus aluminium-oxide and the $\mathrm{S}$ star broad features, pointing to a different dust composition for these $\mathrm{S}$ stars. In the following we concentrate on comparing the $\mathrm{S}$ star spectra with O-rich stars that exhibit low dust columns, since these O-rich stars show the structured $10 \mu \mathrm{m}$ emission complex. The stars that have higher mass-loss rates show profiles close to the classical silicate emission (e.g. Heras \& Hony 2005). See TY Dra in Fig. 1 for an example of such a classical silicate feature.

We readdress the issue of the spectral appearance of the 8-22 $\mu \mathrm{m}$ dust spectra of the $\mathrm{S}$ stars using the available SWS spectra. We choose to restrict ourselves to the relatively small sample of SWS spectra for several reasons. The first reason is to have a consistent data set. More importantly, the sensitivity and spectral resolving power allow us to discuss details not available in the LRS spectra or in many of the ground-based spectra. As an example we mention R Aql for which Speck et al. (2000) report a broad feature without detectable substructure based on their CGS3 spectra, while the SWS spectra clearly resolve the substructures at 9.7, 11 and $13 \mu \mathrm{m}$. Finally, the much wider wavelength coverage allows us to better separate the different molecular and dust contributions. For example, the $10 \mu \mathrm{m}$ region feature of the Mira variable RR Per resembles the S star spectra. However, the complete SWS spectrum reveals very strong molecular absorption and emission bands. This spectrum could have been misinterpreted, in case only the $8-22 \mu \mathrm{m}$ range would had been available.

Kraemer et al. (2002) have classified the ISO/SWS spectra according to the shape of the continuum and dust features. Within their classification $\pi^{1}$ Gru, W Aql and many O-rich stars belong to the same class exhibiting the broad feature due to silicate and aluminium-oxide. Although the peak position and wavelength extend of the emission bump are indeed very similar, the O-rich sources show more substructure with often a prominent sharp emission maximum at $13 \mu \mathrm{m}$ and always substructure at 9.7 and $\sim 11 \mu \mathrm{m}$ (e.g. Cami 2002, see also Fig. 5). This substructure is not found in the spectra of $\pi^{1}$ Gru and W Aql.

A careful survey of all evolved star spectra with sufficient signal to noise in the SWS database yields 9 more sources with a broadened and smooth $\sim 10 \mu \mathrm{m}$ emission feature. These sources are listed in Table 1 and their spectra are shown in Fig. 1. The main conclusion we draw is that such a broad and smooth emission feature is only found in the $\mathrm{M}$ super-giant and the $\mathrm{S}$ star spectra, there are no $O$-rich $A G B$ stars that exhibit the same emission feature. This agrees very well with the findings of Speck et al. (2000) that the broad feature in the AGB stars differs from the broad features found in the super-giants. The ISO spectra show that in fact the broad O-rich AGB star features are always due to a mixture of the $9.7,11$ and $13 \mu \mathrm{m}$ bands in which the $11 \mu \mathrm{m}$ band dominates. Admittedly the substructures are sometimes more pronounced than in other cases which leaves open the possibility that part of the emission in this region, even in the O-rich AGB stars spectra, is due to the same smooth feature present in the S stars. $\chi$ Cyg is the only S star that has exhibits these substructures although the predominant contribution might still be a smooth underlying feature.

Note that the sample of M super-giants displayed in Fig. 1 largely overlaps with the broad featured M super-giants presented by Speck et al. (2000) and consists mostly of super-giants located in the $\mathrm{h}$ and $\chi$ Per association. These sources are by no means typical for all galactic $M$ super-giants. As we already pointed out, the large majority of studied M super-giants exhibits a classical silicate emission feature (Sloan \& Price 1998; Speck et al. 2000). Moreover, seven out of the nine broad featured $\mathrm{M}$ super-giants in our sample reveal UIR emission bands, which are not commonly found in the M super-giant spectra 
(Sylvester et al. 1994, 1998). Sylvester et al. (1998) suggest that this could be related to peculiar elemental abundances at the stellar surfaces.

Another striking aspect of these spectra as a group is the relative strength of the $10 \mu \mathrm{m}$ feature compared to the $18 \mu \mathrm{m}$ feature. In the spectra of the O-rich stars, the $10 \mu \mathrm{m}$ emission is accompanied by an emission feature near 18-20 $\mu \mathrm{m}$, where one would expect the $\mathrm{Si}-\mathrm{O}$ bending modes of the silicates to be present (see Fig. 5). This emission band is systematically weak in the M super-giant and S star spectra in Fig. 1. In some of the sources we find no evidence of the $18 \mu \mathrm{m}$ band at all and three sources exhibit a weak emission band near $15 \mu \mathrm{m}$ instead.

\subsection{Feature extraction}

The differences in the observed features as discussed above are relatively subtle. In order to enhance the contrast we have removed the "continuum" from the spectra. We stress that the continuum as such does not have any clear physical meaning in this context as the same material(s) that give rise to the features contribute to this continuum. It represents only a means to remove those contributions that do not give rise to spectral signatures. It further allows us to compare the relative strength of the remaining bands to other components. Because of the wide range of properties of the stars in the sample - in terms of optical depth, strength of the molecular bands and the excess - we have opted to use a crude method for continuum estimation. The continuum is represented by a sum of a warm black-body-function $\left(F_{v}=B_{v}(T)\right.$; the reddened stellar photosphere) and a cooler modified black-body-function $\left(F_{v}=v^{p} \times\right.$ $B_{v}(T)$; the dust excess). These two functions are fitted simultaneously to selected "continuum-points". The latter are difficult to define unambiguously and we choose to use the following ranges: $2.9-3.35 \mu \mathrm{m}$, when the molecular absorption is not too prominent; $3.35-3.8 \mu \mathrm{m} ; 7-7.5^{\dagger} \mu \mathrm{m} ; 8.5-9.5^{\dagger} \mu \mathrm{m} ; 14-15 \mu \mathrm{m}$; $22-25 \mu \mathrm{m}$ and $36-44 \mu \mathrm{m}$. The ranges marked with a dagger often do not exhibit clear continuum character. These regions have still been included, albeit with a reduced weight, to account for the fact that the continuum should run close to them.

The resulting residual spectra have been co-added to reduce the noise. The co-addition has been applied in two bins, depending on the peak-position of the " $10 " \mu \mathrm{m}$ emission feature. The results are shown in Fig. 6, where we compare the S star profiles to the features found in O-rich stars. Note that the comparison is done on the basis of the extracted feature and does not follow strictly the silicate-index classification. We find that both S-star SEa and SEb classes compare best with the O-rich SEa class and the S-star SEc class with the O-rich SEb class. This mismatch reflects the fact that the underlying continua differs systematically - the O-rich stars being bluer - between both groups of sources (see also below).

Figure 6 demonstrates that indeed the O-rich star exhibit a " $10 " ~ \mu \mathrm{m}$ feature which is composed of three distinct components while the $\mathrm{S}$ star spectra exhibit only a single broad emission band - indicative of a different dust composition between the two groups. There are several other "properties" of these dust spectra that change in league with the differences in dust composition. The lack of the $19.5 \mu \mathrm{m}$ band in the $\mathrm{S}$ star spectra is pronounced. In Fig. 7 we summarise the derived properties of the $\mathrm{S}$ stars compared to those of the M stars. The main conclusion is that the studied $\mathrm{S}$ stars as a sample are redder and exhibit a stronger excess than the O-rich stars with the broad $10 \mu \mathrm{m}$ feature. This indicates the presence of more dust along the line of sight towards the $\mathrm{S}$ stars. There is no significant difference in

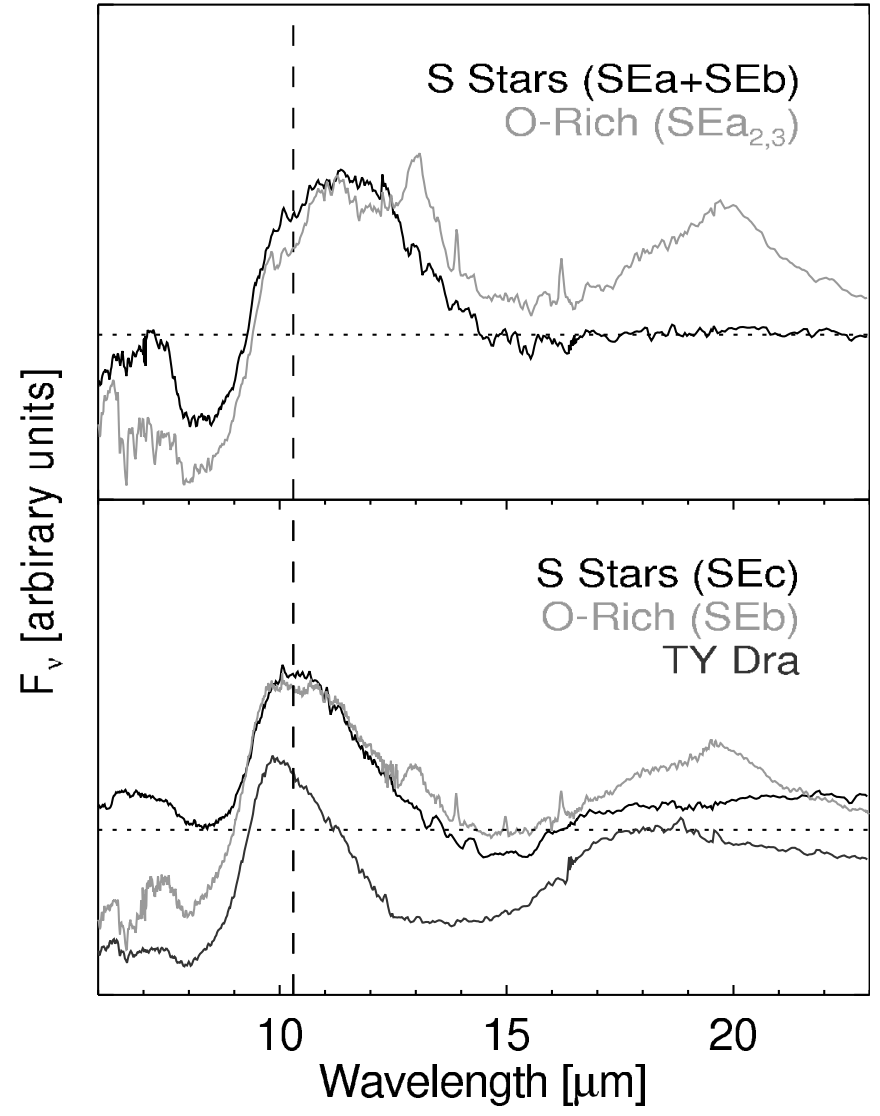

Fig. 6. Comparison of the mean profiles of the $\mathrm{S}$ stars and the O-rich stars. The samples have been split in two parts. Those with profiles peaking beyond $10.5 \mu \mathrm{m}$ (top panel) and those that peak at shorter wavelengths (bottom panel). The dashed line indicates the position $(10.3 \mu \mathrm{m})$ where the mean profiles differ significantly. For reference we also show the feature spectrum of TY Dra, which shows a classical silicate emission feature. The spectrum has been offset for clarity.

the strength of the $10 \mu \mathrm{m}$ feature relative to the dust continuum (Fig. 7c).

\section{Discussion}

As discussed above, the spectral appearance of S stars is significantly different from the O-rich AGB stars. Here we explore the possible explanations for these differences, that is, the relative strength of the emission bands and the displacement of the $10 \mu \mathrm{m}$ feature. The amount of mid-IR excess is probably not directly related to the dust composition. Perhaps the observed difference in dust composition and the differences in the quantities of dust share a common origin in the particular condition that prevail during the $\mathrm{S}$ star evolutionary state.

There are several effects that, if at work, will influence the spectral appearance of dust features even arising from grains with the same chemical composition. The most notable are dust temperature and grain-size. Using the amorphous olivine $\left(\mathrm{Mg}_{0.8} \mathrm{Fe}_{1.2} \mathrm{SiO}_{4}\right)$ that explains (part of) the emission of the O-rich stars well (Heras \& Hony 2005)), we find that the temperature required to explain the observed S star 10-to- $18 \mu \mathrm{m}$ ratio (ignoring the mismatch in peak-position) needs to be higher than $2000 \mathrm{~K}$. And even at such high temperatures (well above the evaporation temperature of silicates) the feature at $18 \mu \mathrm{m}$ would still be too prominent for the most extreme cases, e.g. $\pi^{1}$ Gru or 


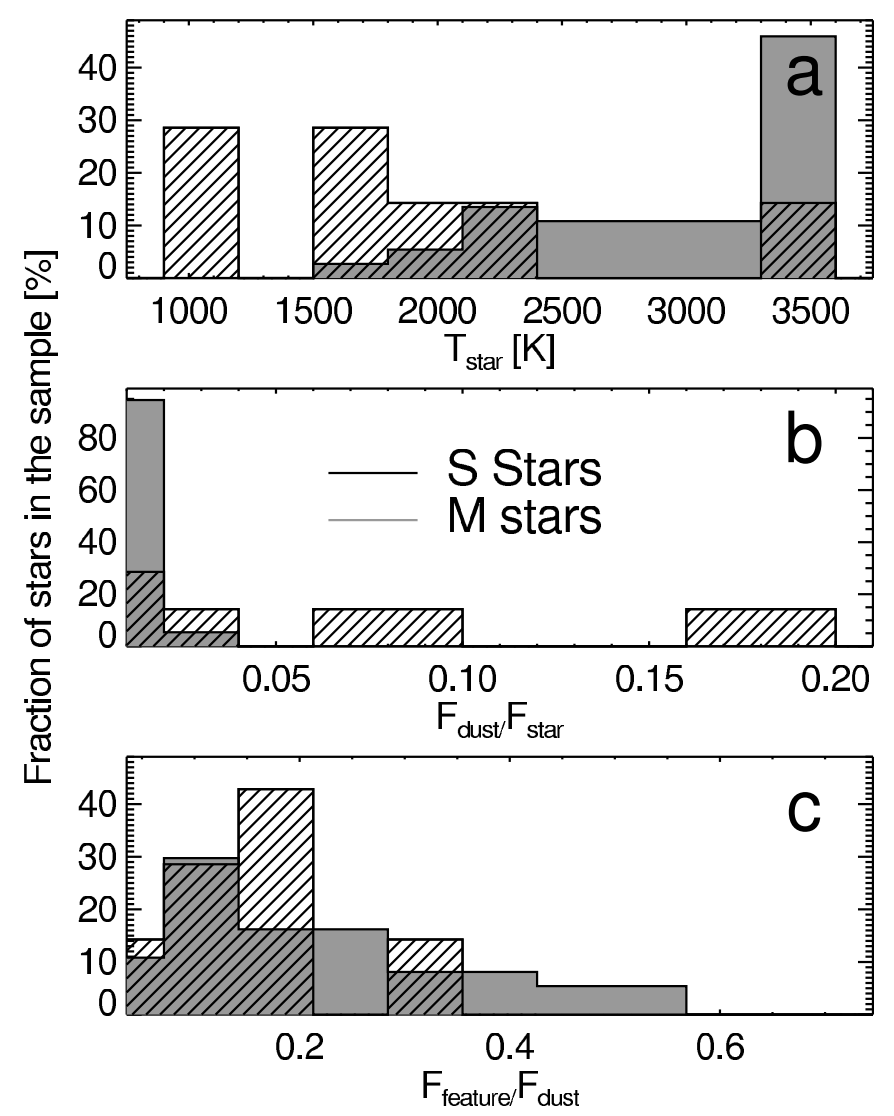

Fig. 7. Properties of the M and S stars. From top to bottom: the fitted temperature of the (reddened) stars; the ratio of the excess to the stellar flux and the ratio of the flux in the feature to the flux in the dust excess. The $\mathrm{S}$ stars in the sample are clearly cooler (i.e. more extincted) and show more excess than the M stars.

W Aql. Thus, dust temperature is excluded as the cause of the weak $18-20 \mu \mathrm{m}$ features.

Grain-size: we have simulated the effects of grain-size on the spectral features of silicates based on simulated optical properties using a Mie calculation (Bohren \& Huffman 1983). The effect of increasing the grain-size from 0.01 to $1 \mu \mathrm{m}$ is indeed a displacement of the $10 \mu \mathrm{m}$ emission feature, but the effect is subtle and it moves the peak by less than $0.3 \mu \mathrm{m}$. This is not enough to explain the $\mathrm{S}$ star spectra. Moreover the ratio of the 10 to $18 \mu \mathrm{m}$ band is only slightly affected. The $18 \mu \mathrm{m}$ band does not shift significantly but becomes somewhat stronger. The S star spectra exhibit an $18 \mu \mathrm{m}$ feature which is little pronounced and peaks at shorter wavelength. We conclude that the grain-size is not the dominant factor for explaining the $\mathrm{S}$ star silicate feature.

The paper by Jäger et al. (2003) is very interesting in terms of the compositional influence on the silicate emission. These authors have studied the IR transmission spectra of nonstoichiometric magnesium-rich silicates. They find that there is a significant shift of the $10 \mu \mathrm{m}$ Si-O stretching resonance as a function of the $\mathrm{Mg}$ to $\mathrm{SiO}_{4}$ ratio. The trend is such that as the $\mathrm{Mg}$ to $\mathrm{SiO}_{4}$ ratio increases, the $10 \mu \mathrm{m}$ peak shifts to longer wavelength, the $18 \mu \mathrm{m}$ band broadens and weakens compared to the continuum and shifts to shorter wavelength. This mimics to a large extent the behaviour observed in the $\mathrm{S}$ star spectra. It should be noted that although the $10 \mu \mathrm{m}$ feature in the laboratory spectra shifts by a large amount $(>1 \mu \mathrm{m})$, it is not completely sufficient to explain the full range observed in the stellar spectra. If the observed shift is indeed linked to the presence of non-stoichiometric silicates, than there could be an obvious connection to the photospheric abundances since the $\mathrm{Mg}$ to $\mathrm{SiO}_{4}$ ratio in the silicate might measure the $\mathrm{Mg}$ to free $\mathrm{O}$ or $\mathrm{SiO}$ in the gas phase. In case of $\mathrm{C} / \mathrm{O} \sim 1$ there is more $\mathrm{Mg}$ in relative terms and those non-stoichiometric silicates may form.

Moreover, the M-super-giants that we present are special not just in their $10 \mu \mathrm{m}$ spectrum but also because they exhibit a dual chemistry with both silicates and PAHs. How this dual chemistry comes about is at present unclear. This may be evidence of the presence of a disk plus wind geometry, shocked regions in the outflow or an abundance pattern in the outflow, which permit this dual chemistry to exist. In any case, it is clear that in some region of the circumstellar environment the chemistry should be close to a $\mathrm{C} / \mathrm{O}$ around unity.

It should be stressed that we cannot draw general conclusions about the dust formation around $\mathrm{S}$ stars from such a small number of sources. We have successfully proposed to observe 90 S stars with IRS Houck et al. (2004) on Spitzer (GO-30737, PI. Hony). The main findings of the current paper, i.e., the presence of the $\mathrm{MgS}$ feature and the shifted $10 \mu \mathrm{m}$ feature, are borne out by the spectra obtained in the Spitzer sample (Smolders et al., in prep). Because of the larger sample the range of spectral features, in particular in the $10-20 \mu \mathrm{m}$ region is larger than what is presented here.

\section{Conclusions}

We have presented a detailed study of dust spectra of the S stars observed by ISO/SWS. The spectra exhibit several unique dust characteristics. In particular, we find two $S$ stars $\left(\pi^{1}\right.$ Gru and W Aql) that exhibit a weak "30" $\mu \mathrm{m}$ feature due to MgS grains. RX Lac and AA Cyg exhibit features between 20-40 $\mu$ m which may be related to Diopside, although this is at most a tentative identification. The $10 \mu \mathrm{m}$ region of the dust-producing $\mathrm{S}$ stars stands out due to its smooth and broad emission feature. The feature is located clearly at longer wavelengths than the classical silicate feature, without showing the characteristic substructure found in the spectra of the O-rich AGB stars with a broad $10 \mu \mathrm{m}$ feature. The peculiar $10 \mu \mathrm{m}$ features of the $\mathrm{S}$ stars are accompanied by very weak or absent features near $18 \mu \mathrm{m}$. We conclude that $S$ stars make different types of dust than the O-rich AGB stars, including those that exhibit shifted $10 \mu \mathrm{m}$ features. As a group the S stars and their dust shells do not compare well to the O-rich AGB stars with structured features, the $\mathrm{S}$ stars are redder and produce more dust. The common explanation for the shifted $10 \mu \mathrm{m}$ band, i.e. a mixture of classical silicate with aluminium-oxide, does not appear to apply to the $\mathrm{S}$ stars. We have explored possible origins of the peculiar spectra. We find that non-stoichiometric silicates with an increased $\mathrm{Mg}$ to $\mathrm{SiO}_{4}$ ratio might be at the origin of the displaced emission. Interestingly, the properties of the peculiar 10 and $20 \mu \mathrm{m}$ features are shared with a small subgroup of $M$ super-giants. These super-giants, which are preferentially found in the $h$ and $\chi$ Per associations, also exhibit the UIR emission features, again strengthening the possible link with the abundances in the dust forming regions.

Acknowledgements. $\mathrm{IA}^{3}$ is a joint development of the SWS consortium. Contributing institutes are SRON, MPE, KUL and the ESA Astrophysics Division. This work was supported by the Dutch ISO Data Analysis Center(DIDAC). The DIDAC is sponsored by SRON, ECAB, ASTRON and the universities of Amsterdam, Groningen, Leiden and Leuven. 


\section{References}

Blanco, V. M. 1955, ApJ, 122, 434

Bohren, C. F., \& Huffman, D. R. 1983, Absorption and scattering of light by small particles (New York: Wiley)

Buscombe, W. 1998, VizieR Online Data Catalog, 3206, 0

Buscombe, W. 2001, VizieR Online Data Catalog, 3222, 0

Cami, J. 2002, Ph.D. Thesis, University of Amsterdam

Chen, P. S., \& Kwok, S. 1993, ApJ, 416, 769

Clegg, P. E., Ade, P. A. R., Armand, C., et al. 1996, A\&A, 315, L38

de Graauw, T., Haser, L. N., Beintema, D. A., et al. 1996, A\&A, 315, L49

Egan, M. P., \& Sloan, G. C. 2001, ApJ, 558, 165

Ferrarotti, A. S., \& Gail, H.-P. 2002, A\&A, 382, 256

Gillett, F. C., Low, F. J., \& Stein, W. A. 1968, ApJ, 154, 677

Hackwell, J. A. 1972, A\&A, 21, 239

Heras, A. M., \& Hony, S. 2005, A\&A, 439, 171

Houck, J. R., Roellig, T. L., Van Cleve, J., et al. 2004, American Astronomical Society Meeting Abstracts, 204

Humphreys, R. M. 1970, AJ, 75, 602

Jäger, C., Dorschner, J., Mutschke, H., Posch, T., \& Henning, T. 2003, A\&A, 408, 193

Kessler, M. F., Steinz, J. A., Anderegg, M. E., et al. 1996, A\&A, 315, L27

Kharchenko, N. V. 2001, Kinematika i Fizika Nebesnykh Tel, 17, 409

Koike, C., Tsuchiyama, A., Shibai, H., et al. 2000, A\&A, 363, 1115
Kraemer, K. E., Sloan, G. C., Price, S. D., \& Walker, H. J. 2002, ApJS, 140, 389 Little-Marenin, I. R., \& Little, S. J. 1988, ApJ, 333, 305

Little-Marenin, I. R., \& Little, S. J. 1990, AJ, 99, 1173

Lorenz-Martins, S., \& Pompeia, L. 2000, MNRAS, 315, 856

Miyata, T., Kataza, H., Okamoto, Y., Onaka, T., \& Yamashita, T. 2000, ApJ, 531, 917

Onaka, T., de Jong, T., \& Willems, F. J. 1989, A\&A, 218, 169

Posch, T., Kerschbaum, F., Mutschke, H., et al. 1999, A\&A, 352, 609

Skinner, C. J., Griffin, I., \& Whitmore, B. 1990, MNRAS, 243, 78

Sloan, G. C., \& Price, S. D. 1995, ApJ, 451, 758

Sloan, G. C., \& Price, S. D. 1998, ApJS, 119, 141

Sloan, G. C., Kraemer, K. E., Price, S. D., \& Shipman, R. F. 2003, ApJS, 147, 379

Solf, J. 1978, A\&AS, 34, 409

Speck, A. K., Barlow, M. J., Sylvester, R. J., \& Hofmeister, A. M. 2000, A\&AS, 146,437

Stephenson, C. B. 1994, VizieR Online Data Catalog, 3168, 0

Stephenson, C. B. 1995, VizieR Online Data Catalog, 3060, 0

Sylvester, R. J. 1999, MNRAS, 309, 180

Sylvester, R. J., Barlow, M. J., \& Skinner, C. J. 1994, MNRAS, 266, 640

Sylvester, R. J., Skinner, C. J., \& Barlow, M. J. 1998, MNRAS, 301, 1083

Winfrey, S., Barnbaum, C., Morris, M., \& Omont, A. 1994, BAAS, 26, 1382

Woolf, N. J., \& Ney, E. P. 1969, ApJ, 155, L181

Yang, X. H., Chen, P., Wang, J., \& He, J. 2007, A\&A, 463, 663 